\title{
Functional Connectivity Delineates Distinct Roles of the Inferior Frontal Cortex and Presupplementary Motor Area in Stop Signal Inhibition
}

\author{
Jeng-Ren Duann, ${ }^{1}$ Jaime S. Ide, ${ }^{2} \mathrm{Xi}$ Luo, ${ }^{2,3}$ and Chiang-shan Ray $\mathrm{Li}^{2,4,5}$ \\ ${ }^{1}$ Swartz Center for Computational Neuroscience, Institute of Neural Computation, University of California, San Diego, San Diego, California 92093, and \\ Departments of ${ }^{2}$ Psychiatry, ${ }^{3}$ Statistics, and ${ }^{4}$ Neurobiology and ${ }^{5}$ Interdepartmental Neuroscience Program, Yale University, New Haven, Connecticut 06519
}

\begin{abstract}
The neural basis of motor response inhibition has drawn considerable attention in recent imaging literature. Many studies have used the go/no-go or stop signal task to examine the neural processes underlying motor response inhibition. In particular, showing greater activity during no-go (stop) compared with go trials and during stop success compared with stop error trials, the right inferior prefrontal cortex (IFC) has been suggested by numerous studies as the cortical area mediating response inhibition. Many of these same studies as well as others have also implicated the presupplementary motor area (preSMA) in this process, in accord with a function of the medial prefrontal cortex in goal-directed action. Here we used connectivity analyses to delineate the roles of IFC and preSMA during stop signal inhibition. Specifically, we hypothesized that, as an integral part of the ventral attention system, the IFC responds to a stop signal and expedites the stop process in the preSMA, the primary site of motor response inhibition. This hypothesis predicted that preSMA and primary motor cortex would show functional interconnectivity via the basal ganglia circuitry to mediate response execution or inhibition, whereas the IFC would influence the basal ganglia circuitry via connectivity with preSMA. The results of Granger causality analyses in 57 participants confirmed this hypothesis. Furthermore, psychophysiological interaction showed that, compared with stop errors, stop successes evoked greater effective connectivity between the IFC and preSMA, providing additional support for this hypothesis. These new findings provided evidence critically differentiating the roles of IFC and preSMA during stop signal inhibition and have important implications for our understanding of the component processes of inhibitory control.
\end{abstract}

\section{Introduction}

The go/no-go and stop signal task (SST) have been widely used to investigate the behavioral and neural processes of motor response inhibition (Logan and Cowan, 1984). In these behavioral tasks, a "go" stimulus required participants to respond within a time window. Because these go trials occur most of the time, they set up a prepotent response tendency. In contrast, the stop signal instructs participants to withhold their response. The rationale is that, when response inhibition is in place, participants are able to stop after seeing the stop signal. Thus, many previous studies have compared stop success (SS) with stop error (SE) trials or simply stop trials with go trials and identified bilateral or right inferior prefrontal cortex (IFC) as a cortical site of inhibitory motor control (Verbruggen and Logan, 2008). It was theorized that the IFC projects to the subthalamic nucleus (STN) in a hyper-direct pathway for motor inhibitory control (Aron and Poldrack, 2006).

\section{Received March 17, 2009; revised July 6, 2009; accepted July 11, 2009.}

This study was supported by National Institutes of Health Grants R01DA023248 (C.-s.R.L.) and Physician Scientist Training Grant K12DA000167 (Bruce Rounsaville). We thank Olivia Hendrick and Sarah Bednarski for editing this manuscript.

Correspondence should be addressed to Dr. Chiang-shan Ray Li, Connecticut Mental Health Center, S103, Department of Psychiatry, Yale University School of Medicine, 34 Park Street, New Haven, CT 06519. E-mail: chiang-shan.li@yale.edu.

DOI:10.1523/JNEUROSCI.1300-09.2009

Copyright $\odot 2009$ Society for Neuroscience $\quad$ 0270-6474/09/2910171-09\$15.00/0
Many of these and other studies have also isolated the presupplementary motor area (preSMA) as a key locus of response inhibition, in keeping with a role of this medial prefrontal structure in action control and selection (Nachev et al., 2008). In particular, greater preSMA activation was associated with shorter stop signal reaction time (SSRT), an index of inhibitory control as computed on the basis of the race model (Li et al., 2006a). An important question is thus whether the IFC and preSMA play a similar or different role in motor response inhibition.

The extensive literature has suggested that the IFC is part of the ventral attention system, which activates in response to the detection of a salient target, particularly when the target is behaviorally relevant (Linden et al., 1999; Corbetta and Shulman, 2002; Bledowski et al., 2004; Corbetta et al., 2008; Hampshire et al., 2009). For instance, in spatial cueing paradigms, the right IFC along with the temporal parietal junction responds and reorients attention to an external stimulus that occurs unexpectedly or infrequently, when the stimulus is a target (Kincade et al., 2005; Serences et al., 2005). In the stop signal task, the stop signal is both infrequent and behaviorally relevant. Thus, greater IFC activity during stop compared with go trials may simply reflect attentional processing of the stop signal. By increasing activity in response to the stop signal, the IFC may serve to orient attention and resources to the stop process and, as a result, facilitate stop signal inhibition.

The current study aimed to substantiate these roles of the IFC and preSMA in stop signal inhibition. We hypothesized that the 
IFC would facilitate stop signal inhibition via functional connectivity with the preSMA and sought to confirm this hypothesis with Granger causality analysis (GCA). Specifically, we predicted that the preSMA and primary motor cortex (PMC) would show strong interconnectivity with the basal ganglia circuitry of motor control to determine the outcome of go and stop processes, whereas the IFC would indirectly influence the basal ganglia circuitry via connectivity with preSMA. We also predicted that, in psychophysiological interaction (PPI) (Friston et al., 1997; Gitelman et al., 2003), stop success would evoke greater effective connectivity between the IFC and preSMA compared with stop error trials.

\section{Materials and Methods}

Subjects and behavioral task. Sixty subjects (30 men, 22-45 years of age, all right-handed) were paid to participate in the study. All subjects signed a written consent after details of the study were explained, in accordance to institute guidelines and procedures approved by the Yale Human Investigation Committee.

We used a simple reaction time (RT) task in this stop signal paradigm (Logan and Cowan, 1984; Li et al., 2006a, 2008a,b). There were two trial types: "go" and "stop," randomly intermixed. A small dot appeared on the screen to engage attention at the beginning of a go trial. After a randomized time interval (fore period) between 1 and $5 \mathrm{~s}$, the dot turned into a circle (the "go" signal), which served as an imperative stimulus, prompting the subjects to quickly press a button. The circle vanished at a button press or after $1 \mathrm{~s}$ had elapsed, whichever came first, and the trial was terminated. A premature button press before the appearance of the circle also terminated the trial. Three-quarters of all trials were go trials. The remaining one-quarter were stop trials. In a stop trial, an additional "X," the "stop" signal, appeared after and replaced the go signal. The subjects were told to withhold button press after seeing the stop signal. Likewise, a trial terminated at button press or when $1 \mathrm{~s}$ had elapsed since the appearance of the stop signal. The stop signal delay (SSD) — the time interval between the go and stop signal—started at $200 \mathrm{~ms}$ and varied from one stop trial to the next according to a staircase procedure: if the subject succeeded in withholding the response, the SSD increased by 64 ms; conversely, if they failed, SSD decreased by $64 \mathrm{~ms}$ (Levitt, 1971; De Jong et al., 1990). There was an intertrial interval of $2 \mathrm{~s}$. Subjects were instructed to respond to the go signal quickly while keeping in mind that a stop signal could come up in a small number of trials. Before the functional magnetic resonance imaging (fMRI) study each subject had a practice session outside the scanner. In the scanner, each subject completed four 10-min runs of the task with the SSD updated manually across runs. Depending on the actual stimulus timing (trials varied in fore-period duration) and speed of response, the total number of trials varied slightly across subjects in an experiment. With the staircase procedure, we anticipated that the subjects would succeed in withholding their response in approximately half of the stop trials.

We computed a critical SSD that represents the time delay between go and stop signals that would be required for a subject to succeed in 50\% of the stop trials (Levitt, 1971). Specifically, SSDs across trials were grouped into runs, with each run being defined as a monotonically increasing or decreasing series. We derived a mid-run estimate by taking the middle SSD (or average of the two middle SSDs if there was an even number of SSDs) of every second run. The critical SSD was computed by taking the mean of all mid-run SSDs. It was reported that, except for experiments with a small number of trials $(<30)$, the mid-run estimate was close to the maximum likelihood estimate of $X_{50}$ (50\% positive response, i.e., $50 \%$ SS in the SST) (Wetherill et al., 1966). The SSRT was computed by subtracting the critical SSD from the median go trial RT (Logan, 1994).

Thirty subjects were also imaged in a $10 \mathrm{~min}$ "resting state" session, in which they were instructed to stay awake and relaxed, with their eyes closed.

Imaging protocol. Conventional $\mathrm{T}_{1}$-weighted spin echo sagittal anatomical images were acquired for slice localization using a $3 \mathrm{~T}$ scanner (Siemens Trio). Anatomical images of the functional slice locations were next obtained with spin echo imaging in the axial plane parallel to the anterior commissure-posterior commissure (AC-PC) line with the following parameters: repetition time (TR), $300 \mathrm{~ms}$; echo time (TE), $2.5 \mathrm{~ms}$; bandwidth, $300 \mathrm{~Hz} /$ pixel; flip angle, $60^{\circ}$; field of view, $220 \times 220 \mathrm{~mm}$; matrix, $256 \times 256$; 32 slices with slice thickness, $4 \mathrm{~mm}$ and no gap. Functional, blood oxygenation level-dependent (BOLD) signals were then acquired with a single-shot gradient echo echoplanar imaging (EPI) sequence. Thirty-two axial slices parallel to the AC-PC line covering the whole brain were acquired with the following parameters: TR, $2000 \mathrm{~ms}$; TE, $25 \mathrm{~ms}$; bandwidth, $2004 \mathrm{~Hz} /$ pixel; flip angle, $85^{\circ}$; field of view, $220 \times$ $220 \mathrm{~mm}$; matrix, $64 \times 64$; 32 slices with slice thickness, $4 \mathrm{~mm}$ and no gap. Three hundred images were acquired in each run for a total of four runs.

Spatial preprocessing and general linear modeling. Data were analyzed with Statistical Parametric Mapping version 5 (SPM5) (Wellcome Department of Imaging Neuroscience, University College London, London, UK). Images from the first five TRs at the beginning of each trial were discarded to enable the signal to achieve steady-state equilibrium between response function pulsing and relaxation. Images of each individual subject were first corrected for slice timing, realigned (motioncorrected), and unwarped (Andersson et al., 2001; Hutton et al., 2002). A mean functional image volume was constructed for each subject for each run from the realigned image volumes. These mean images were normalized to an MNI (Montreal Neurological Institute) EPI template with affine registration, followed by nonlinear transformation (Friston et al., 1995a; Ashburner and Friston, 1999). The normalization parameters determined for the mean functional volume were then applied to the corresponding functional image volumes for each subject. Finally, images were smoothed with a Gaussian kernel of $10 \mathrm{~mm}$ at full-width at half-maximum.

Statistical modeling of the imaging data was described in detail in our previous studies (Li et al., 2006a, 2008a,b). Briefly, four main types of trial outcome were distinguished: go success, go error, SS, and SE. An analytical statistical design was constructed for each individual subject, using the general linear model with the onsets of go signal in each of these trial types convolved with a canonical hemodynamic response function (HRF) and with the temporal derivative of the canonical HRF entered as regressors in the model (Friston et al., 1995b). Realignment parameters in all six dimensions were also entered in the model. The data were high-pass filtered ( $128 \mathrm{~s}$ cutoff) to remove low-frequency signal drifts. Serial autocorrelation was corrected by a first-degree autoregressive, or AR(1), model. In the first-level analysis, we constructed for each individual subject a contrast between SS and SE. The "con" or contrast (difference in $\beta$ ) images of the first-level analysis were then used for the second-level group statistics (random effect analysis) (Penny and Holmes, 2004). Brain regions were identified using an atlas (Duvernoy, 1999). All templates are in MNI space, and voxel activations are presented in MNI coordinates. We used MarsBaR to derive for each individual subject the effect size of activity change for regions of interest (ROIs) (Brett et al., 2002) (http://marsbar.sourceforge.net/).

Granger causality analysis. Task-related and resting state time series were examined with GCA of multivariate autoregressive models (Granger, 1969), a method widely used to describe "causal" influence between sets of EEG or fMRI time series (Ding et al., 2000; Kamiński et al., 2001; Goebel et al., 2003; Kuś et al., 2004; Roebroeck et al., 2005; Deshpande et al., 2009; Sato et al., 2009). In this analysis, we included as ROIs the preSMA, right IFC (rIFC), and PMC, caudate head, and the STN. The masks of preSMA, rIFC, and PMC were derived on the basis of regional brain activations obtained by Li et al. (2006a). The MNI coordinates of these three structures were as follows: $x=-4, y=36, z=56$ (preSMA); $x=44, y=48, z=-12$ (rIFC); and $x=-36, y=-8, z=52$ (PMC). We included in the model the left caudate head, which showed greater activation in association with short stop signal reaction time (Li et al., 2008b). Masks of the left caudate head and the STN were obtained from the anatomical automatic labeling atlas (TzourioMazoyer et al., 2002).

The application of multivariate autoregressive modeling requires that each ROI time series is covariance stationary, which we examined with the Augmented Dickey Fuller (ADF) test (Hamilton, 1994). ADF test verified that there is no unit root in the time series. BOLD time series were concatenated across all four sessions for each individual subject. 
The data of 57 of the 60 subjects were covariance stationary and subjected to GCA.

The preprocessed BOLD time series were averaged for each subject across all voxels in each of the five ROIs. In a multidimensional vector autoregressive (VAR) model (Goebel et al., 2003; Sato et al., 2006; Seth and Edelman, 2007), we computed the Granger causality (G-causality) between the time series

$$
\begin{aligned}
{\left[\begin{array}{c}
x_{1, t} \\
x_{2, t} \\
\vdots \\
x_{5, t}
\end{array}\right]=\left[\begin{array}{c}
\mu_{1} \\
\mu_{2} \\
\vdots \\
\mu_{5}
\end{array}\right]+} & {\left[\begin{array}{cccc}
A_{11}^{1} & A_{12}^{1} & \cdots & A_{15}^{1} \\
A_{21}^{1} & A_{22}^{1} & \cdots & A_{25}^{1} \\
\vdots & \vdots & \ddots & \vdots \\
A_{51}^{1} & A_{52}^{1} & \cdots & A_{55}^{1}
\end{array}\right]\left[\begin{array}{c}
x_{1, t-1} \\
x_{2, t-1} \\
\vdots \\
x_{5, t-1}
\end{array}\right]+\cdots } \\
& +\left[\begin{array}{cccc}
A_{11}^{k} & A_{12}^{k} & \cdots & A_{15}^{k} \\
A_{21}^{k} & A_{22}^{k} & \cdots & A_{25}^{k} \\
\vdots & \vdots & \ddots & \vdots \\
A_{51}^{k} & A_{52}^{k} & \cdots & A_{55}^{k}
\end{array}\right]\left[\begin{array}{c}
x_{1, t-k} \\
x_{2, t-k} \\
\vdots \\
x_{5, t-k}
\end{array}\right]+\left[\begin{array}{c}
\mu_{1} \\
\mu_{2} \\
\vdots \\
\mu_{5}
\end{array}\right]
\end{aligned}
$$

assuming that

$$
\sum_{u}=\left[\begin{array}{cccc}
\sum_{11} & \sum_{12} & \cdots & \sum_{15} \\
\sum_{21} & \sum_{22} & \cdots & \sum_{25} \\
\vdots & \vdots & \ddots & \vdots \\
\sum_{51} & \sum_{52} & \cdots & \sum_{55}
\end{array}\right]
$$

In Equation $1, x_{i, t}, i=1,2, \ldots, 5$ represent the time series of IFC, preSMA, PMC, caudate head, and STN, respectively, with $x_{i, t}$ and $x_{i, t-p}$ representing the value of the time series at time $t$ and time $t-p$, respectively, and $p=1,2, \ldots, k$, where $k$ is the order of the VAR model. The optimal time lag was determined using Akaike information criterion (Akaike, 1974). Also, $\mu_{i}(i=1,2, \ldots, 5)$ are the means of the five time series and $A_{i, j}^{p}$, where $i, j=1,2, \ldots, 5$, and $p=1,2, \ldots, k$, are the linear coefficients of the VAR model (i.e., the contributions of each "lagged" observation to the predicted values of $\left.\mathrm{x}_{i, t}\right) \cdot u_{i}(i=1,2, \ldots, 5)$ are the residuals (prediction errors) for each of the time series, which were assumed to have a Gaussian distribution, $N\left(0, \Sigma_{u}\right)$ (Eq. 2).

Time series $x_{1}$ is said to be "Granger-caused" (or G-caused) by time series $x_{2}$ if the inclusion of time series $x_{2}$ reduces the variance of the residual $\left(\Sigma_{12}\right.$ in Eq. 2) obtained by the autoregressive model of time series $x_{1}$ itself $\left(\Sigma_{11}\right)$ (Granger, 1969). We tested the significance of the G-causality between time series $x_{1}$ and $x_{2}$ by an $F$ test:

$$
S=\frac{\left(\sum_{i=1}^{T} \sum_{11}^{i}-\sum_{i=1}^{T} \sum_{12}^{i}\right) / p}{\sum_{i=1}^{T} \sum_{12}^{i} /(T-2 p-1)} \sim F_{p, T-2 p-1},
$$

where $T$ is the total number of time points, and $p$ is the order of the VAR model. If the test statistic of Equation 3 was greater than a specified significance criterion (e.g., $p<0.0025$, correcting for a total of 20 comparisons for each subject), we rejected the null hypothesis that time series $x_{2}$ did not G-cause time series $x_{1}$ (Geweke, 1982). Note that it is not necessary that time series $x_{1}$ and $x_{2}$ have reciprocal G-causality. The direction of G-causality between time series $x_{1}$ and $x_{2}$ is determined by the residual variance $\Sigma_{12}$ and $\Sigma_{21}$, and these two terms may not be identical because they are derived by two different regression estimations.

Because we used a multidimensional VAR model, we could determine all the residual terms in a single model and, importantly, identified whether there was an intermediate node between two target nodes. That is, a multidimensional model helped differentiate the G-causality between $\mathrm{X} \rightarrow \mathrm{Y}$ and $\mathrm{X} \rightarrow \mathrm{Z} \rightarrow \mathrm{Y}$, which would be identical if a bivariate approach was used (Geweke, 1982).

We determined the G-causalities of the five time series for individual subjects, correcting for multiple comparisons $(p<0.05 / 20=0.0025)$, and computed the significance of the effective connectivity for the entire sample using a binomial test. In an alternative analysis, we determined the G-causalities for individual subjects by bootstrapping from the data time series of the five ROIs, before group analysis with the binomial test

\begin{tabular}{|c|c|c|c|c|c|}
\hline & \multicolumn{5}{|c|}{ EFFECT } \\
\hline & IFC & PMC & presMA & Caudate & STN \\
\hline \multicolumn{6}{|l|}{ CAUSE } \\
\hline IFC & & 28 & 48 & 24 & 19 \\
\hline PMC & 34 & & 37 & 49 & 50 \\
\hline PreSMA & 37 & 24 & & 48 & 23 \\
\hline Caudate & 24 & 24 & 29 & & 33 \\
\hline STN & 26 & 45 & 32 & 40 & \\
\hline IFC & & 2 & 5 & 5 & 3 \\
\hline PMC & 2 & & 3 & 6 & 8 \\
\hline PreSMA & 3 & 2 & & 4 & 4 \\
\hline Caudate & 3 & 2 & 2 & & 6 \\
\hline STN & 3 & 8 & 2 & 11 & \\
\hline
\end{tabular}
(see below).
Table 1. Number of subjects showing significant Granger causality: stop signal task (top) and resting state (bottom) time series

Binomial test: $n>38$ for $p<0.01$ and $n>36$ for $p<0.05$, task data $(n=57) ; n>21$ for $p<0.01$ and $n>19$ for $p<0.05$, resting state data $(n=30)$.

Psychophysiological interaction. PPI describes how functional connectivity between brain regions is altered as a result of psychological context or variables (Friston et al., 1997; Gitelman et al., 2003). In pursuit of our hypothesis that the IFC is functionally connected with the preSMA to expedite stop signal inhibition, we anticipated greater connectivity between the two brain regions during SS compared with SE trials.

The time series of the first eigenvariate of the BOLD signal were temporally filtered, mean corrected, and deconvolved to generate the time series of the neuronal signal for the source region-the IFC-as the physiological variable in the PPI. The psychological variable represented the contrast between SS and SE trials: SS - SE. An additional regressor represented the interaction between the psychological and physiological factors. These regressors were convolved with the canonical HRF and entered into the regression model. The interaction term in the resulting SPM showed areas with significant differential connectivity to the IFC because of the psychological context SS - SE. PPI analysis was performed for each subject, and the resulting images of contrast estimates were used for random effect group analysis.

\section{Results}

\section{Stop signal performance}

Subjects had a mean go trial success rate of $96.1 \pm 4.2 \%$ (mean \pm $\mathrm{SD}$, across subjects) with a median RT of $557 \pm 120 \mathrm{~ms}$. The average stop success rate was $50.5 \pm 2.4 \%$, suggesting that their performance was adequately tracked by the staircase procedure. The average stop signal reaction time was $205 \pm 38 \mathrm{~ms}$, well in the range of the values reported in numerous previous studies (Tseng and Li, 2008).

\section{Regional brain activations during stop signal performance}

We examined regional brain activation associated with stop signal inhibition, using the same analyses as in our previous studies (Li et al., 2006a, 2008c). The results of the current cohort of 57 subjects confirmed our previous findings. Compared with SE, SS trials evoked greater activation in bilateral superior/middle and inferior frontal cortices. In contrast, compared with SS, SE trials evoked greater activation in many cortical and subcortical structures, including the dorsal anterior cingulate cortex (ACC) and the thalamus (supplemental Fig. 1, available at www.jneurosci.org as supplemental material). Furthermore, on the basis of a median split of the SSRT, we compared 28 subjects with short with the other 28 with long SSRT ( $174 \pm 23$ vs $235 \pm$ $28 \mathrm{~ms} ; p<0.0001)$ and observed greater activation in a dorsomedial region of the superior frontal cortex and a subregion in the rostral ACC (supplemental Fig. 2, available at www.jneurosci.org as supplemental material). 


\section{Granger causality analysis}

The results of GCA of the stop signal task time series showed that the preSMA and PMC have significant connectivity with the caudate head and STN and that the IFC projected to the preSMA but not to the basal ganglia $(p<0.0025$, corrected for multiple comparisons, for individual GCA; $p<0.01$, binomial test for group analysis) (Table 1, top; Fig. 1). With $p<$ 0.05 , the binomial test for group results showed that the IFC and preSMA are reciprocally connected. In contrast, no significant Granger causality was observed for any of the connections for the resting state time series (Table 1, bottom).

To further confirm these results, we used GCA on time series resampled (bootstrapped) from our data. In essence, for individual subjects, by resampling 2500 times from the data time series in each ROI, we created surrogate time series with the same mean, variance, autocorrelation function, and power spectrum as the data time series (Theiler et al., 1992; Kamiński et al., 2001; Kuś et al., 2004; Deshpande et al., 2009). The resulting $F$ values from GCA on these surrogate time series constituted the null hypothesis, which was tested against the data time series. G-causality was considered significant at $p<0.05$, corrected for false discovery rate, for individual connections (Genovese et al., 2002). Significance of G-causality was determined at the group level with a binomial test. The results confirmed connectivity between PMC as well as preSMA and the subcortical circuitry and the interconnectivity between the IFC and preSMA (Fig. 2). Furthermore, the IFC did not show G-causality with the caudate or STN in either direction.

\section{Psychophysiological interaction}

Figure 3 shows the brain regions that demonstrated greater connectivity with the IFC during SS compared with SE trials, at a threshold of $p<0.005$, uncorrected and 10 voxels in the extent of activation. These brain regions included bilateral superior temporal, inferior frontal, and visual cortices, as well as a dorsomedial region in the superior frontal cortex (Table 2). To test our hypothesis specifically, we performed a region of interest analysis focusing on the preSMA with small volume correction. The results showed a significant cluster in the ROI: $x=0, y=36, z=56$; $p<0.05$, corrected for family-wise error of multiple comparisons. At the same threshold (uncorrected $p<0.005$ and 10 voxels), a small cluster located in the region of ventromedial prefrontal cortex ( $x=16, y=48, z=-4$; voxel $Z=3.20,15$ voxels $)$ showed a negative PPI.

In a previous study, we showed greater preSMA activity in association with short compared with long SSRT (Li et al., 2006). Thus, to examine whether this functional connectivity between IFC and preSMA differs with respect to SSRT, we compared the effect sizes of this connectivity between subjects with short and long SSRT, on the basis of a median split ( $174 \pm 23$ vs $235 \pm 28$ ms; $p<0.0001 ; n=28$ in each group). The results showed that the two groups did not differ in IFC-preSMA connectivity: $0.53 \pm 0.93$ vs $0.31 \pm 0.98(p=0.217$, one-tailed two-sample $t$

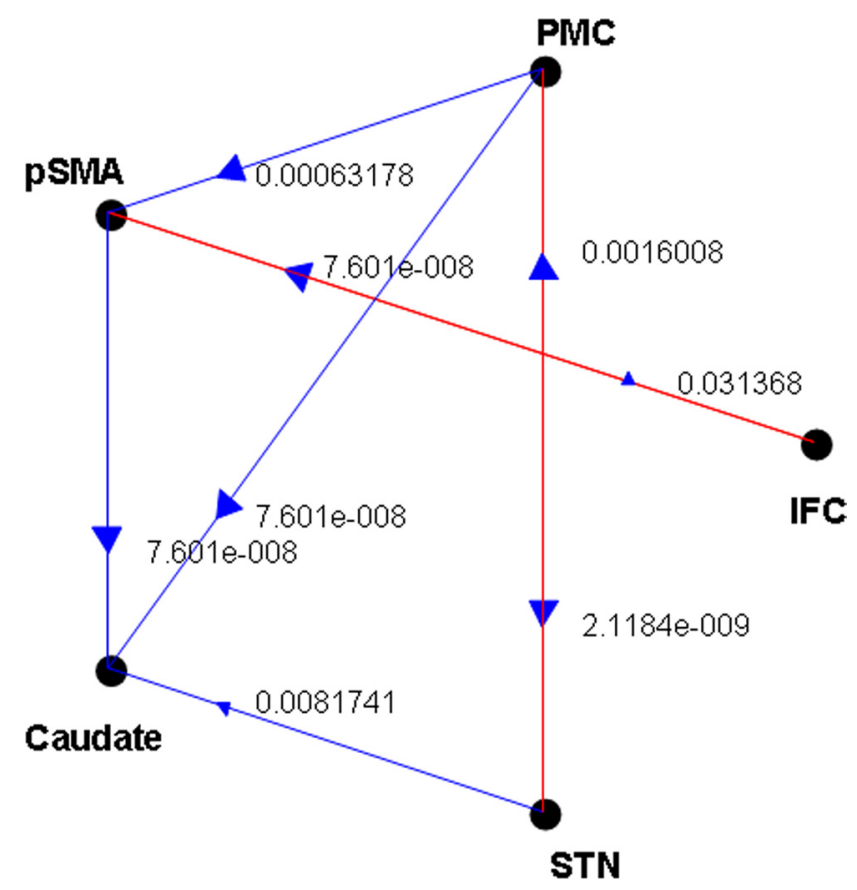

Figure 2. The results of $\mathrm{G}$-causality analysis with significance of individual connectivity tested against bootstrapped surrogate time series. $p$ values are obtained from binomial test. Overall, the pattern of $\mathrm{G}$-causality was almost identical to that shown in Figure 1.

test). Comparison between subjects in the first and last quartiles of SSRT $(157 \pm 19$ vs $254 \pm 29 \mathrm{~ms} ; p<0.0001 ; n=14$ in each group) yielded negative results: $0.30 \pm 1.15$ vs $0.67 \pm 1.02$ ( $p=$ 0.199 , one-tailed two-sample $t$ test). We also failed to observe a correlation between the effect size of the IFC-preSMA connec- 


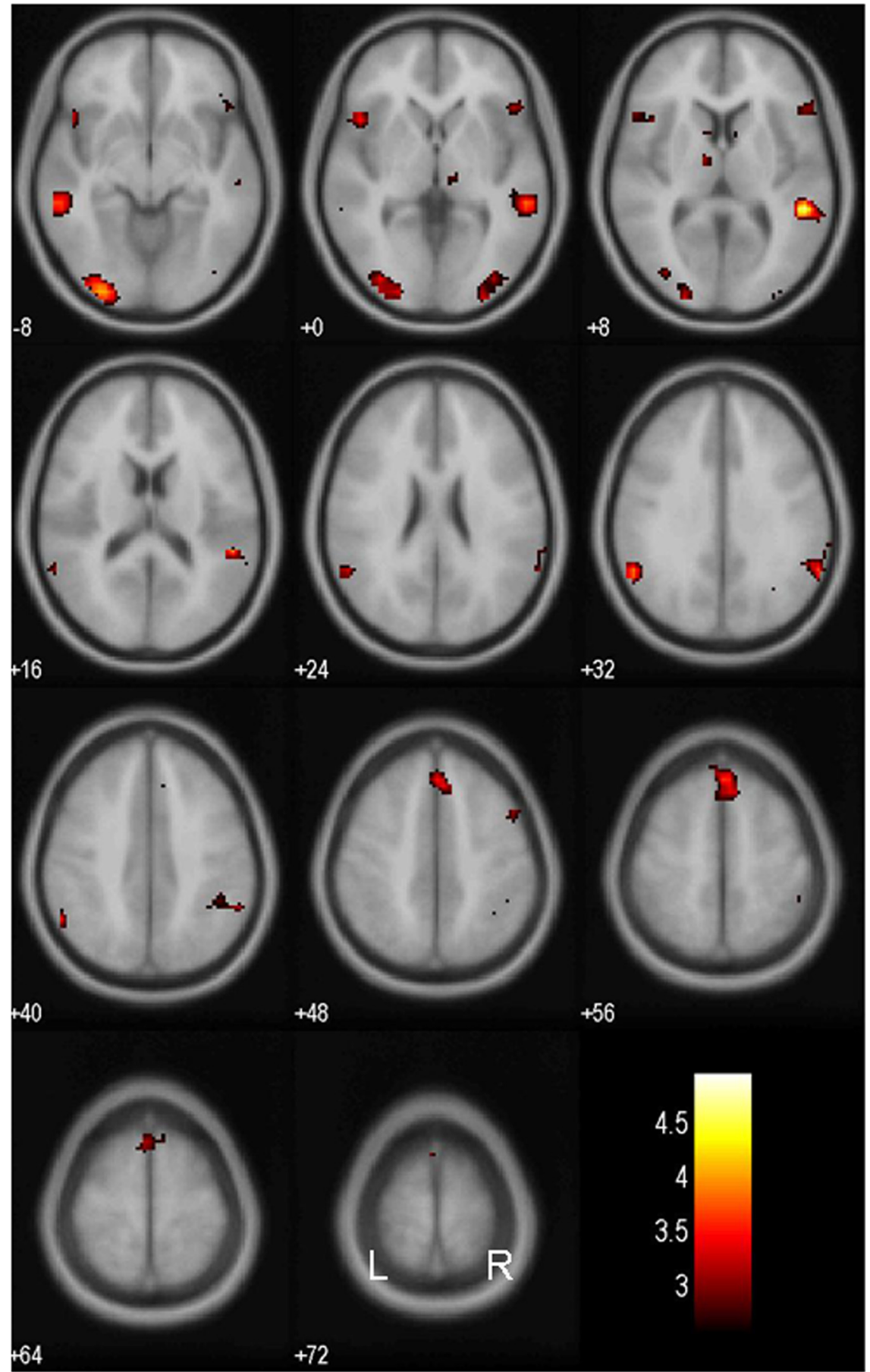

Figure 3. Brain regions showing greater PPI with the inferior frontal cortex during stop success compared with stop error trials. BOLD contrast was overlaid on a T1 structural image in axial sections. Neurological orientation: right = right. Color bar represents voxel $T$ value. L, Left; R, right.

tivity with SSRT across all 57 subjects $(r=0.053 ; p=0.688$, Pearson's regression).

\section{Discussion}

Our current findings from the Granger causality analyses showed that the PMC and preSMA are functionally connected with the caudate head and STN. Furthermore, the IFC is connected with the preSMA but not the caudate head or STN. Thus, with strong interconnectivity with the basal ganglia circuitry of motor control, the PMC and preSMA are in a position to engage the com petition of go and stop processes, whereas the IFC indirectly influence the basal ganglia circuitry via projection to the preSMA. These new findings provide evidence differentiating the roles of the IFC and preSMA during stop signal inhibition. In particular, these data are inconsistent with the hypothesis of a hyperdirect pathway from the IFC to STN for motor inhibitory control (Aron and Poldrack, 2006).

The results from PPI analyses further corroborated this hypothesis: the IFC showed greater connectivity with the preSMA during stop success than during stop error trials. A number of other brain regions including the superior temporal and inferior frontal gyri as well as the visual cortices also showed a significant positive PPI. Although these findings were not specifically related to our hypothesis, they were consistent with many studies implicating these temporal/parietal structures in awareness and attentional binding of perceptual inputs (Driver and Mattingley, 1998; Linden, 2005; Campanella and Belin, 2007; Decety and Lamm, 2007; Redcay, 2008). Greater functional connectivity with temporal/ parietal structures also appeared to be in accord with the relatively common finding of parietal activation in the literature of the no/no-go and stop signal task (Menon et al., 2001; Rubia et al., 2001; Garavan et al., 2002; Jaffard et al., 2008; Karch et al., 2009). Greater connectivity with the visual cortices may underlie a mechanism of attentional enhancement of visual information processing (Brefczynski and DeYoe, 1999; Smith et al., 2000; Slotnick et al., 2003), and parietal activation might be the source of this striate cortical modulation (Poghosyan et al., 2005).

In previous reports, we demonstrated greater preSMA but not IFC activation (during stop success $>$ stop error) in association with short compared with long SSRT (Li et al., 2006a; Chao et al., 2009). One question is whether the PPI between the IFC and preSMA is related to SSRT. Neither group-based comparison nor linear correlation showed a significant association between IFC-preSMA connectivity and SSRT. These results suggested that, although the IFC serves to detect the stop signal, the process of response suppression likely does not occur until the signal reaches the preSMA. This preliminary finding thus seems to further demarcate the roles of the IFC and preSMA during stop signal inhibition.

As described previously, the IFC is part of the ventral attention system, which activates to the detection of a salient, behaviorally relevant target (for review, see Corbetta and Shulman, 2002; Corbetta et al., 2008). In the stop signal task, the stop signal is both salient, because it is less frequent, and behaviorally relevant, because it demands a change of response. Thus, the saliency pro- 
Table 2. Brain regions showing positive PPI

\begin{tabular}{|c|c|c|c|c|c|c|}
\hline \multirow[b]{2}{*}{ Cluster size (voxels) } & \multirow[b]{2}{*}{ Voxel $Z$ value } & \multicolumn{3}{|c|}{ MNI coordinate $(\mathrm{mm})$} & \multirow[b]{2}{*}{ Sidedness } & \multirow[b]{2}{*}{ Identified brain region } \\
\hline & & $x$ & $y$ & $z$ & & \\
\hline 64 & 4.45 & 52 & -40 & 12 & $\mathrm{R}$ & Superior/transverse temporal gyrus \\
\hline 76 & 3.76 & -32 & -92 & -8 & $\mathrm{~L}$ & Occipital cortex \\
\hline 35 & 3.63 & -56 & -56 & 36 & $\mathrm{~L}$ & Angular gyrus \\
\hline 78 & 3.60 & -4 & 16 & 68 & $\mathrm{~L}$ & Superior frontal gyrus \\
\hline 39 & 3.46 & 60 & -52 & 32 & $\mathrm{R}$ & Angular gyrus \\
\hline 21 & 3.45 & -60 & -36 & -8 & L & Superior/transverse temporal gyrus \\
\hline 30 & 3.37 & -48 & 16 & -4 & L & Inferior frontal gyrus or anterior insula \\
\hline 25 & 3.16 & 40 & 32 & -4 & $\mathrm{R}$ & Inferior frontal gyrus \\
\hline 25 & 3.08 & 32 & -96 & 4 & $\mathrm{R}$ & Occipital cortex \\
\hline
\end{tabular}

$p<0.005$, uncorrected, and 10 voxels in spatial extent. L, Left; $R$, right.

cessing of the stop signal may explain greater IFC activation during stop (or no-go) trials compared with go trials (Garavan et al., 1999; Konishi et al., 1999; Liddle et al., 2001; Rubia et al., 2003, 2005; Aron and Poldrack, 2006; Chevrier et al., 2007; Leung and Cai, 2007; Xue et al., 2008). Many other studies have also provided evidence supporting a role of the IFC in target detection (Bledowski et al., 2004; Linden, 2005; Hampshire et al., 2009). In particular, Hampshire and colleagues showed that the IFC responds to target stimuli even when they were equated in frequency to the distractor stimuli, ruling out a surprise or "oddball" effect. Furthermore, by probing response only at the end of the stimulus sequence, the investigators demonstrated that response suppression was not required for this IFC activity to be observed. Thus, as suggested by Hampshire and colleagues, these findings support a role of the IFC in target detection during planned responses, in accord with the current results.

A recent study by Chikazoe et al. (2009) attempted to distinguish oddball from response inhibition activity by introducing infrequent go trials during a go/no-go task. They showed greater response in a posterior locus of the inferior frontal cortex during no-go compared with infrequent go trials and suggested that this area is specifically related to response suppression. Conversely, compared with an infrequent go response, a no-go response (no response) would likely require greater attentional processing to be successfully executed. For instance, one might speculate that, although lapses of attention during no-go trials would prevent the stop process from being initiated in time, resulting in a no-go error, similar lapses during infrequent go trials would perhaps simply delay the go process. Thus, by contrasting successful no-go and infrequent go trials, one might be isolating neural processes directly related to attention. Nonetheless, the studies of Chikazoe and colleagues are interesting because they delineated inferior frontal subregions specialized for different aspects of go/ no-go performance (Chikazoe et al., 2008; Hirose et al., 2009).

How might one isolate the neural correlates of response inhibition during the stop signal task, independent of such attentionrelated activity? Previous work of Logan and Cowan provided a useful approach (Logan and Cowan, 1984; Logan, 1994). Logan and Cowan hypothesized in a model that the "go" and "stop" processes race to finish. The go process prepares and generates the movement, whereas the stop process inhibits movement initiation: whichever process finishes first determines whether a response will be initiated or not. Importantly, the go and stop processes race toward the activation threshold independently. Thus, the time required for the stop signal to be processed so a response is withheld (i.e., SSRT) can be computed on the basis of the go trial RT distribution and the odds of successful inhibits for different time delays between the go and stop signals. This is achieved by estimating the "critical" SSD at which a response can be correctly stopped in $\sim 50 \%$ of the stop trials and subtracting the critical SSD from the median go trial RT (Logan, 1994). Generally speaking, the SSRT is the time required for a subject to cancel the movement after seeing the stop signal. Studies have used changes in SSRT as an index of the development of inhibitory control across lifespan (Williams et al., 1999; Bedard et al., 2002). A longer SSRT indicates poor response inhibition, and the wide behavioral literature of the stop signal task has used prolonged SSRT as an index of impaired motor inhibitory control in patients with neurological or psychiatric conditions (Kooijmans et al., 2000; Rieger et al., 2003; Gauggel et al., 2004; Bekker et al., 2005; Bellgrove et al., 2006; Li et al., 2006b; Alderson et al., 2007; Sagaspe et al., 2007; Huddy et al., 2009; Huizenga et al., 2009; McAlonan et al., 2009).

Notably, our previous work suggested that the preSMA activity is inversely associated with the SSRT in individuals who did not differ in any other aspects of the stop signal performance (Li et al., 2006a; Chao et al., 2009). This preSMA activity in inhibitory control is consistent with many previous studies suggesting functions of goal-directed action in this medial cortical structure (Shima et al., 1996; Boecker et al., 1998, 2008; Rushworth et al., 2002; Lau et al., 2004; Nachev et al., 2005, 2007; Brass and Haggard, 2007; de Jong and Paans, 2007; Leung and Cai, 2007; Mueller et al., 2007; Sumner et al., 2007; Simmonds et al., 2008; Suskauer et al., 2008). For instance, patients with preSMA lesions were impaired in inhibiting ongoing movements without showing changes in simple reaction time (Nachev et al., 2007). Such a role of preSMA in inhibitory motor control was also supported by electrophysiological studies. Stuphorn and Schall (2006) showed that subthreshold electrical microstimulation of the presupplementary eye field improves inhibitory function (i.e., shortening SSRT) in macaque monkeys performing the stop signal task. Electrical stimulation in the pre-SMA suppressed an automatic unwanted action but boosted a controlled desired action in macaque monkeys performing a "saccade-overriding" task (Isoda and Hikosaka, 2007).

Together, the current findings from GCA and PPI analyses suggested that both the IFC and preSMA are involved but play different roles during stop signal inhibition, with the IFC mediating attentional processing of the stop signal and the preSMA mediating motor inhibitory control. GCA has been a useful tool in describing effective connectivity between brain regions during fMRI of a cognitive task (Roebroeck et al., 2005; Abler et al., 2006; Stilla et al., 2007; Deshpande et al., 2008; Upadhyay et al., 2008). In particular, without a priori assumptions about the network connectivity, GCA is well suited for hypothesis testing. The present study set out to differentiate two hypotheses, with one postulating direct connectivity between the IFC and STN and the other postulating a projection from the IFC to preSMA, which is 
connected with the basal ganglia circuitry. Our results clearly favored the latter hypothesis. Conversely, one is cautioned against over-interpreting the patterns of connectivity. For instance, the current results could not be used to specify the individual roles of caudate nucleus and STN during stop signal inhibition.

To summarize, the current findings are inconsistent with the hypothesis of a hyperdirect pathway from the IFC to basal ganglia for inhibitory motor control. The results suggest that the IFC and preSMA play different roles in stop signal inhibition, with the IFC mediating attentional processing of the stop signal and the preSMA mediating response inhibition. The current findings have important implications for our understanding of the component processes of inhibitory control. In particular, deficits in stop signal inhibition have been implicated in many clinical conditions, including attention deficit hyperactivity disorder and Parkinson's disease (Bush et al., 2005; McCloskey et al., 2005; Li and Sinha, 2008). These results would facilitate our understanding of the source of inhibitory control deficits in these illnesses.

\section{References}

Abler B, Roebroeck A, Goebel R, Höse A, Schönfeldt-Lecuona C, Hole G, Walter H (2006) Investigating directed influences between activated brain areas in a motor-response task using fMRI. Magn Reson Imaging 24:181-185.

Akaike H (1974) A new look at the statistical model identification. IEEE Trans Auto Control 19:716-723.

Alderson RM, Rapport MD, Kofler MJ (2007) Attention-deficit/hyperactivity disorder and behavioral inhibition: a meta-analytic review of the stopsignal paradigm. J Abnorm Child Psychol 35:745-758.

Andersson JL, Hutton C, Ashburner J, Turner R, Friston K (2001) Modeling geometric deformations in EPI time series. Neuroimage 13:903-919.

Aron AR, Poldrack RA (2006) Cortical and subcortical contributions to Stop signal response inhibition: role of the subthalamic nucleus. J Neurosci 26:2424-2433.

Ashburner J, Friston KJ (1999) Nonlinear spatial normalization using basis functions. Hum Brain Mapp 7:254-266.

Bedard AC, Nichols S, Barbosa JA, Schachar R, Logan GD, Tannock R (2002) The development of selective inhibitory control across the life span. Dev Neuropsychol 21:93-111.

Bekker EM, Overtoom CC, Kenemans JL, Kooij JJ, De Noord I, Buitelaar JK, Verbaten MN (2005) Stopping and changing in adults with ADHD. Psychol Med 35:807-816.

Bellgrove MA, Chambers CD, Vance A, Hall N, Karamitsios M, Bradshaw JL (2006) Lateralized deficit of response inhibition in early-onset schizophrenia. Psychol Med 36:495-505.

Bledowski C, Prvulovic D, Goebel R, Zanella FE, Linden DE (2004) Attentional systems in target and distractor processing: a combined ERP and fMRI study. Neuroimage 22:530-540.

Boecker H, Dagher A, Ceballos-Baumann AO, Passingham RE, Samuel M, Friston KJ, Poline J, Dettmers C, Conrad B, Brooks DJ (1998) Role of the human rostral supplementary motor area and the basal ganglia in motor sequence control: investigations with $\mathrm{H}_{2}{ }^{15} \mathrm{O}$ PET. J Neurophysiol 79:1070-1080.

Boecker H, Jankowski J, Ditter P, Scheef L (2008) A role of the basal ganglia and midbrain nuclei for initiation of motor sequences. Neuroimage 39:1356-1369.

Brass M, Haggard P (2007) To do or not to do: the neural signature of self-control. J Neurosci 27:9141-9145.

Brefczynski JA, DeYoe EA (1999) A physiological correlate of the "spotlight" of visual attention. Nat Neurosci 2:370-374.

Brett M, Anton JL, Valabregue R, Poline JP (2002) Region of interest analysis using an SPM toolbox. Abstract presented at the 8th International Conference on Functional Mapping of the Human Brain, June 2-6, Sendai, Japan.

Bush G, Valera EM, Seidman LJ (2005) Functional neuroimaging of attention-deficit/hyperactivity disorder: a review and suggested future directions. Biol Psychiatry 57:1273-1284.

Campanella S, Belin P (2007) Integrating face and voice in person perception. Trends Cogn Sci 11:535-543.
Chao HH, Luo X, Chang JL, Li CS (2009) Greater activation of the anterior pre-supplementary motor area but not the inferior frontal cortex in association with short stop signal reaction time: an intrasubject analysis. BMC Neurosci 10:75.

Chevrier AD, Noseworthy MD, Schachar R (2007) Dissociation of response inhibition and performance monitoring in the stop signal task using event-related fMRI. Hum Brain Mapp 28:1347-1358.

Chikazoe J, Jimura K, Asari T, Yamashita K, Morimoto H, Hirose S, Miyashita Y, Konishi S (2009) Functional dissociation in right inferior frontal cortex during performance of go/no-go task. Cereb Cortex 19:146-152.

Corbetta M, Shulman GL (2002) Control of goal-directed and stimulusdriven attention in the brain. Nat Rev Neurosci 3:201-215.

Corbetta M, Patel G, Shulman GL (2008) The reorienting system of the human brain: from environment to theory of mind. Neuron 58:306-324.

de Jong BM, Paans AM (2007) Medial versus lateral prefrontal dissociation in movement selection and inhibitory control. Brain Res 1132:139-147.

De Jong R, Coles MG, Logan GD, Gratton G (1990) In search of the point of no return: the control of response processes. J Exp Psychol Hum Percept Perform 16:164-182.

Decety J, Lamm C (2007) The role of the right temporoparietal junction in social interaction: how low-level computational processes contribute to meta-cognition. Neuroscientist 13:580-593.

Deshpande G, Hu X, Stilla R, Sathian K (2008) Effective connectivity during haptic perception: a study using Granger causality analysis of functional magnetic resonance imaging data. Neuroimage 40:1807-1814.

Deshpande G, LaConte S, James GA, Peltier S, Hu X (2009) Multivariate Granger causality analysis of fMRI data. Hum Brain Mapp 30:1361-1373.

Ding M, Bressler SL, Yang W, Liang H (2000) Short-window spectral analysis of cortical event-related potentials by adaptive multivariate autoregressive modeling: data preprocessing, model validation, and variability assessment. Biol Cybern 83:35-45.

Driver J, Mattingley JB (1998) Parietal neglect and visual awareness. Nat Neurosci 1:17-22.

Duvernoy HM (1999) The human brain: surface, blood supply, and threedimensional sectional anatomy, Ed 2. New York: Springer.

Friston KJ, Ashburner J, Frith CD, Polone JB, Heather JD, Frackowiak RS (1995a) Spatial registration and normalization of images. Hum Brain Mapp 2:165-189.

Friston KJ, Holmes AP, Worsley KJ, Poline JB, Frith CD, Frackowiak RSJ (1995b) Statistical parametric maps in functional imaging: a general linear approach. Hum Brain Mapp 2:189-210.

Friston KJ, Buechel C, Fink GR, Morris J, Rolls E, Dolan RJ (1997) Psychophysiological and modulatory interactions in neuroimaging. Neuroimage 6:218-229.

Garavan H, Ross TJ, Stein EA (1999) Right hemispheric dominance of inhibitory control: an event-related functional MRI study. Proc Natl Acad Sci U S A 96:8301-8306.

Garavan H, Ross TJ, Murphy K, Roche RA, Stein EA (2002) Dissociable executive functions in the dynamic control of behavior: inhibition, error detection, and correction. Neuroimage 17:1820-1829.

Gauggel S, Rieger M, Feghoff TA (2004) Inhibition of ongoing responses in patients with Parkinson's disease. J Neurol Neurosurg Psychiatry 75:539-544.

Genovese CR, Lazar NA, Nichols T (2002) Thresholding of statistical maps in functional neuroimaging using the false discovery rate. Neuroimage $15: 870-878$.

Geweke J (1982) Measurement of linear dependence and feedback between multiple time series. J Am Stat Assoc 77:304-313.

Gitelman DR, Penny WD, Ashburner J, Friston KJ (2003) Modeling regional and psychophysiologic interactions in fMRI: the importance of hemodynamic deconvolution. Neuroimage 19:200-207.

Goebel R, Roebroeck A, Kim DS, Formisano E (2003) Investigating directed cortical interactions in time-resolved fMRI data using vector autoregressive modeling and Granger causality mapping. Magn Reson Imaging 21:1251-1261.

Granger CW (1969) Investigating causal relations by econometric models and cross-spectral methods. Econometrica 37:424-438.

Hamilton JD (1994) Time series analysis. Princeton, NJ: Princeton UP.

Hampshire A, Thompson R, Duncan J, Owen AM (2009) Selective tuning of the right inferior frontal gyrus during target detection. Cogn Affect Behav Neurosci 9:103-112.

Hirose S, Chikazoe J, Jimura K, Yamashita KI, Miyashita Y, Konishi S (2009) 
Sub-centimeter scale functional organization in human inferior frontal gyrus. Neuroimage 47:442-450.

Huddy VC, Aron AR, Harrison M, Barnes TR, Robbins TW, Joyce EM (2009) Impaired conscious and preserved unconscious inhibitory processing in recent onset schizophrenia. Psychol Med 39:907-916.

Huizenga HM, van Bers BM, Plat J, van den Wildenberg WP, van der Molen MW (2009) Task complexity enhances response inhibition deficits in childhood and adolescent attention-deficit/hyperactivity disorder: a meta-regression analysis. Biol Psychiatry 65:39-45.

Hutton C, Bork A, Josephs O, Deichmann R, Ashburner J, Turner R (2002) Image distortion correction in fMRI: a quantitative evaluation. Neuroimage 16:217-240.

Isoda M, Hikosaka O (2007) Switching from automatic to controlled action by monkey medial frontal cortex. Nat Neurosci 10:240-248.

Jaffard M, Longcamp M, Velay JL, Anton JL, Roth M, Nazarian B, Boulinguez P (2008) Proactive inhibitory control of movement assessed by eventrelated fMRI. Neuroimage 42:1196-1206.

Kamiński M, Ding M, Truccolo WA, Bressler SL (2001) Evaluating causal relations in neural systems: granger causality, directed transfer function and statistical assessment of significance. Biol Cybern 85:145-157.

Karch S, Mulert C, Thalmeier T, Lutz J, Leicht G, Meindl T, Möller HJ, Jäger L, Pogarell O (2009) The free choice whether or not to respond after stimulus presentation. Hum Brain Mapp. Advance online publication. Retrieved Jan. 26, 2009. doi:10.1002/hbm.20722.

Kincade JM, Abrams RA, Astafiev SV, Shulman GL, Corbetta M (2005) An event-related functional magnetic resonance imaging study of voluntary and stimulus-driven orienting of attention. J Neurosci 25:4593-4604.

Konishi S, Nakajima K, Uchida I, Kikyo H, Kameyama M, Miyashita Y (1999) Common inhibitory mechanism in human inferior prefrontal cortex revealed by event-related functional MRI. Brain 122:981-991.

Kooijmans R, Scheres A, Oosterlaan J (2000) Response inhibition and measures of psychopathology: a dimensional analysis. Child Neuropsychol 6:175-184.

Kuś R, Kamiński M, Blinowska KJ (2004) Determination of EEG activity propagation: pair-wise versus multichannel estimate. IEEE Trans Biomed Eng 51:1501-1510.

Lau HC, Rogers RD, Haggard P, Passingham RE (2004) Attention to intention. Science 303:1208-1210.

Leung HC, Cai W (2007) Common and differential ventrolateral prefrontal activity during inhibition of hand and eye movements. J Neurosci 27:9893-9900.

Levitt H (1971) Transformed up-down methods in psychoacoustics. J Acoust Soc Am 49:Suppl 2:467+.

Li CS, Sinha R (2008) Inhibitory control and emotional stress regulation: neuroimaging evidence for frontal-limbic dysfunction in psychostimulant addiction. Neurosci Biobehav Rev 32:581-597.

Li CS, Huang C, Constable RT, Sinha R (2006a) Imaging response inhibition in a stop signal task: neural correlates independent of signal monitoring and post-response processing. J Neurosci 26:186-192.

Li CS, Milivojevic V, Kemp K, Hong K, Sinha R (2006b) Performance monitoring and stop signal inhibition in abstinent patients with cocaine dependence. Drug Alcohol Depend 85:205-212.

Li CS, Huang C, Yan P, Paliwal P, Constable RT, Sinha R (2008a) Neural correlates of post-error slowing in a stop signal task. J Cogn Neurosci 20:1021-1029.

Li CS, Yan P, Sinha R, Lee TW (2008b) The subcortical processes of motor response inhibition during a stop signal task. Neuroimage 41:1352-1363.

Li CS, Yan P, Chao HH, Sinha R, Paliwal P, Constable RT, Zhang S, Lee TW (2008c) Error-specific medial cortical and subcortical activity during the stop signal task: a functional magnetic resonance imaging study. Neuroscience 155:1142-1151.

Liddle PF, Kiehl KA, Smith AM (2001) Event-related fMRI study of response inhibition. Hum Brain Mapp 12:100-109.

Linden DE (2005) The p300: where in the brain is it produced and what does it tell us? Neuroscientist 11:563-576.

Linden DE, Prvulovic D, Formisano E, Völlinger M, Zanella FE, Goebel R, Dierks T (1999) The functional neuroanatomy of target detection: an fMRI study of visual and auditory oddball tasks. Cereb Cortex 9:815-823.

Logan GD (1994) On the ability to inhibit thought and action: a user's guide to the stop signal paradigm. In: Inhibitory processes in attention, memory and language (Dagenbach D, Carr TH, eds), pp 189-239. San Diego: Academic.
Logan GD, Cowan WB (1984) On the ability to inhibit thought and action: a theory of an act of control. Psychol Rev 91:295-327.

McAlonan GM, Cheung V, Chua SE, Oosterlaan J, Hung SF, Tang CP, Lee CC, Kwong SL, Ho TP, Cheung C, Suckling J, Leung PW (2009) Agerelated grey matter volume correlates of response inhibition and shifting in attention-deficit hyperactivity disorder. Br J Psychiatry 194:123-129.

McCloskey MS, Phan KL, Coccaro EF (2005) Neuroimaging and personality disorders. Curr Psychiatry Rep 7:65-72.

Menon V, Adleman NE, White CD, Glover GH, Reiss AL (2001) Errorrelated brain activation during a Go/NoGo response inhibition task. Hum Brain Mapp 12:131-143.

Mueller VA, Brass M, Waszak F, Prinz W (2007) The role of the preSMA and the rostral cingulate zone in internally selected actions. Neuroimage 37:1354-1361.

Nachev P, Rees G, Parton A, Kennard C, Husain M (2005) Volition and conflict in human medial frontal cortex. Curr Biol 15:122-128.

Nachev P, Wydell H, O'neill K, Husain M, Kennard C (2007) The role of the pre-supplementary motor area in the control of action. Neuroimage 36 [Suppl 2]:T155-T163.

Nachev P, Kennard C, Husain M (2008) Functional role of the supplementary and pre-supplementary motor areas. Nat Rev Neurosci 9:856-869.

Penny W, Holmes AP (2004) Random-effects analysis. In: Human brain function (Frackowiak RSJ, Ashburner JT, Penny WD, Zeki S, Friston KJ, Frith CD, Dolan RJ, Price CJ, eds), pp 843-850. San Diego: Elsevier.

Poghosyan V, Shibata T, Ioannides AA (2005) Effects of attention and arousal on early responses in striate cortex. Eur J Neurosci 22:225-234.

Redcay E (2008) The superior temporal sulcus performs a common function for social and speech perception: implications for the emergence of autism. Neurosci Biobehav Rev 32:123-142.

Rieger M, Gauggel S, Burmeister K (2003) Inhibition of ongoing responses following frontal, nonfrontal, and basal ganglia lesions. Neuropsychology 17:272-282.

Roebroeck A, Formisano E, Goebel R (2005) Mapping directed influence over the brain using Granger causality and fMRI. Neuroimage 25:230-242.

Rubia K, Russell T, Overmeyer S, Brammer MJ, Bullmore ET, Sharma T, Simmons A, Williams SC, Giampietro V, Andrew CM, Taylor E (2001) Mapping motor inhibition: conjunctive brain activations across different versions of go/no-go and stop tasks. Neuroimage 13:250-261.

Rubia K, Smith AB, Brammer MJ, Taylor E (2003) Right inferior prefrontal cortex mediates response inhibition while mesial prefrontal cortex is responsible for error detection. Neuroimage 20:351-358.

Rubia K, Smith AB, Brammer MJ, Toone B, Taylor E (2005) Abnormal brain activation during inhibition and error detection in medicationnaïve adolescents with ADHD. Am J Psychiatry 162:1067-1075.

Rushworth MF, Hadland KA, Paus T, Sipila PK (2002) Role of the human medial frontal cortex in task switching: a combined fMRI and TMS study. J Neurophysiol 87:2577-2592.

Sagaspe P, Philip P, Schwartz S (2007) Inhibitory motor control in apneic and insomniac patients: a stop task study. J Sleep Res 16:381-387.

Sato JR, Junior EA, Takahashi DY, de Maria Felix M, Brammer MJ, Morettin PA (2006) A method to produce evolving functional connectivity maps during the course of an fMRI experiment using wavelet-based timevarying Granger causality. Neuroimage 31:187-196.

Sato JR, Takahashi DY, Arcuri SM, Sameshima K, Morettin PA, Baccalá LA (2009) Frequency domain connectivity identification: an application of partial directed coherence in fMRI. Hum Brain Mapp 30:452-461.

Serences JT, Shomstein S, Leber AB, Golay X, Egeth HE, Yantis S (2005) Coordination of voluntary and stimulus-driven attentional control in human cortex. Psychol Sci 16:114-122.

Seth AK, Edelman GM (2007) Distinguishing causal interactions in neural populations. Neural Comput 19:910-933.

Shima K, Mushiake H, Saito N, Tanji J (1996) Role for cells in the presupplementary motor area in updating motor plans. Proc Natl Acad Sci U S A 93:8694-8698.

Simmonds DJ, Pekar JJ, Mostofsky SH (2008) Meta-analysis of Go/No-go tasks demonstrating that fMRI activation associated with response inhibition is task-dependent. Neuropsychologia 46:224-232.

Slotnick SD, Schwarzbach J, Yantis S (2003) Attentional inhibition of visual processing in human striate and extrastriate cortex. Neuroimage 19:1602-1611. 
Smith AT, Singh KD, Greenlee MW (2000) Attentional suppression of activity in the human visual cortex. Neuroreport 11:271-277.

Stilla R, Deshpande G, LaConte S, Hu X, Sathian K (2007) Posteromedial parietal cortical activity and inputs predict tactile spatial acuity. J Neurosci 27:11091-11102.

Stuphorn V, Schall JD (2006) Executive control of countermanding saccades by the supplementary eye field. Nat Neurosci 9:925-931.

Sumner P, Nachev P, Morris P, Peters AM, Jackson SR, Kennard C, Husain M (2007) Human medial frontal cortex mediates unconscious inhibition of voluntary action. Neuron 54:697-711.

Suskauer SJ, Simmonds DJ, Fotedar S, Blankner JG, Pekar JJ, Denckla MB, Mostofsky SH (2008) Functional magnetic resonance imaging evidence for abnormalities in response selection in attention deficit hyperactivity disorder: differences in activation associated with response inhibition but not habitual motor response. J Cogn Neurosci 20:478-493.

Theiler J, Eubank S, Longtin A, Galdrikian B, Farmer JD (1992) Testing for nonlinearity in time series: the method of surrogate data. Physica D 58:77-94.
Tseng YC, Li CS (2008) The effects of response readiness and error monitoring on saccade countermanding. Open Psychol J 1:18-25.

Tzourio-Mazoyer N, Landeau B, Papathanassiou D, Crivello F, Etard O, Delcroix N, Mazoyer B, Joliot M (2002) Automated anatomical labeling of activations in SPM using a macroscopic anatomical parcellation of the MNI MRI single-subject. Neuroimage 15:273-289.

Upadhyay J, Silver A, Knaus TA, Lindgren KA, Ducros M, Kim DS, TagerFlusberg H (2008) Effective and structural connectivity in the human auditory cortex. J Neurosci 28:3341-3349.

Verbruggen F, Logan GD (2008) Response inhibition in the stop-signal paradigm. Trends Cogn Sci 12:418-424.

Wetherill GB, Chen H, Vasudeva RB (1966) Sequential estimation of quantal response curves: a new method of estimation. Biometrika 53:439-454.

Williams BR, Ponesse JS, Schachar RJ, Logan GD, Tannock R (1999) Development of inhibitory control across the life span. Dev Psychol 35:205-213.

Xue G, Aron AR, Poldrack RA (2008) Common neural substrates for inhibition of spoken and manual responses. Cereb Cortex 18:1923-1932. 